\title{
De l'intuition primordiale à la pensée consciente et symbolique
}

\section{From primordial intuition to conscious and symbolic thinking}

\author{
MIGUEL ESPINOZA
}

Université de Strasbourg

Recibido: 02/11/2020 Aceptado: 10/02/2021

\section{RESUMÉ}

La philosophie de la nature qui donne sens au problème de la relation entre l'intuition primordiale et la pensée symbolique est un système spéculatif, cohérent et nécessaire, métaphysiquement réaliste et naturaliste. La continuité entre les différentes strates de la nature permet d'imaginer que l'intuition primordiale est un événement situé dans une zone où le physicochimique et la matière vivante s'entrelacent, et de concevoir que de l'intuition primordiale émerge, à son tour, la pensée symbolique.

\section{MOTS-CLES \\ INTUITION PRIMORDIALE, PENSÉE SYMBOLIQUE, CONTINUITÉ NATURELLE, NATURALISME UNIVERSEL}

\begin{abstract}
The philosophy of nature that gives sense to the problem concerning the relationship between primordial intuition and symbolic thinking is a speculative system coherent and necessary, metaphysically realist and naturalist. The continuity between the different strata of nature makes it possible to imagine, first, that primordial intuition takes place in a region where the physicochemical stratum and living matter intertwine, secondly, that symbolic thinking emerges from primordial intuition.
\end{abstract}

\section{KEYWORDS}

PRIMORDIAL INTUITION, SYMBOLIC THINKING, NATURAL CONTINUITY, COMPREHENSIVE NATURALISM 


\section{L'INTELLIGIBILITÉ INTRINSÉQUE}

LA PHILOSOPHIE NATURELLE DÉPLOYÉE dans cet essai, qui donne un sens au problème de la compréhension de l'intuition primordiale et de la naissance de la pensée symbolique, est un système spéculatif, logiquement cohérent et nécessaire, métaphysiquement réaliste et naturaliste. Un vrai problème — qu'il faut distinguer de l'énigme et du mystère - contient la solution en puissance dans son énoncé, et si la nature a des intuitions d'elle-même, si elle se pense, cela veut dire qu'elle possède en elle-même les principes nécessaires à sa propre connaissance. Il y a donc dans cet exposé une relation dynamique, un va-et-vient entre le problème et les principes susceptibles de l'éclairer.

D'après mon réalisme métaphysique et naturaliste la nature est intrinsèquement intelligible et elle est la seule réalité: elle n'est pas l'apparence d'autre chose. Elle est intrinsèquement intelligible parce qu'elle est ordonnée par la nécessité causale. L'intelligibilité n'est pas un cadeau que l'homme, ou un autre monde idéal, font à la réalité. L'ordre et la raison existent dans la nature préconsciente, pré-mentale, animale ou humaine, avant d'exister dans nos appareils psychiques. «Si les théories se rapportent à une intelligence, c'est d'abord à celle qui est immanente aux choses». ${ }^{1}$ Ce réalisme naturaliste est métaphysique. Il n'est pas scientifique parce que ni le réel ni le naturel ne sont déterminés exclusivement par les formalismes et les procédures observationnelles ou expérimentales de la science.

La nature est un réseau causal serré et c'est pourquoi seule la nécessité limite la nécessité. Expliquer, c'est monter dans l'échelle de la nécessité. Comprendre une entité, un processus, signifie se rendre compte qu'ils ne peuvent pas exister autrement, et qu'une fois dans l'existence, ils participent à l'élaboration du réseau causal qui constitue la nature.

Alors, comment la nature a-t-elle une intuition d'elle-même ? L'intelligibilité intrinsèque est une des conditions sine qua non de la réflexivité de la question. Par contre, si la nature avait besoin d'une lumière extranaturelle pour être comprise, alors cette lumière serait inconcevable et ineffable. La description serait construite avec des mots négatifs et vides: extra-naturel, a-causal, im-matériel, non-spatial, a-temporel. Or, avec des termes négatifs, rien de positif ne peut se construire.

Tout ce qui existe devient, et le devenir est la conséquence d'une causalité complexe. L'espace et le temps ne sont pas deux substances séparables de la matière mais deux aspects de la matière en devenir. Les processus et les objets ont une certaine durée et la permanence des objets résulte de la causalité matérielle. Les catégories de la matière en devenir, la causalité, l'espace et le 
temps font partie d'une série de concepts fondamentaux que nous partageons avec l'ensemble des êtres vivants. Les relations intelligibles produites par les relations causales rendent possible non seulement l'intuition primordiale mais, également, l'induction, la déduction et l'ensemble des élaborations symboliques de la conscience. Une chose est désordonnée, irrationnelle ou arrive par hasard seulement par rapport à un sujet qui n'a pas appréhendé les relations intelligibles: en soi, dans la nature, rien n'est désordonné ni n’arrive par hasard.

\section{LA SYMPATHIE NATURELLE}

Le naturalisme de mon système spéculatif est intégral. Les niveaux d'organisation sont, tous, naturels: mathématique, physique, chimique, biologique, psychique et social. Mais j'aimerais faire remarquer que cette façon si tranchée de stratifier la réalité, utile au progrès des sciences particulières, rend difficile le travail du philosophe qui cherche à décrire la continuité naturelle. Rappelons que d'après Aristote, on entend par continu «une sorte de cohérence. Ainsi je dis d'une chose qu'elle est continue quand les limites par lesquelles les deux parties de cette chose se touchent, se sont confondues et réunies, et qu'alors, comme le mot même l'indique, elles se continuent et se tiennent; or, c'est là ce qui ne pourrait avoir lieu tant que les extrémités restent deux et ne s'unissent pas. Évidemment il suit de cette définition qu'il ne peut y avoir de continuité réelle qu'entre des choses qui peuvent, en se touchant, arriver à ne former qu'une seule et même chose naturellement». ${ }^{2}$

De plus cette façon si tranchée de stratifier le réel n'est pas tout à fait vraie. Par exemple, on ne sait pas bien ce qu'est le physique car nous pensons avec des neurones, faits, entre autres, d'électrons, lesquels suivent aussi les lois de la psychologie et, quand nous pensons, ils suivent, entre autres, les lois de la logique. Nous ne savons pas comment la conscience s'intègre au plan de l'organisme, les affections psychosomatiques en témoignent: on peut mourir suite à une mauvaise nouvelle. Il s'ensuit qu'il n'est pas évident que le réductionnisme scientifique, qui essaye d'expliquer le supérieur par l'inférieur, soit plus probable que le holisme métaphysique.

Après avoir souligné l'intelligibilité intrinsèque et le fait que tout le réel est naturel, il faut noter que la nature produit tous les existants avec les mêmes éléments et les mêmes mécanismes de causalité. Cette observation rend possible la sympathie naturelle. En adaptant à notre problème la phrase d'Empédocle: «Le semblable est connu par le semblable», je fais un pas plus loin: l'identique a une intuition de l'identique. Cela signifie qu'il y a

2 Aristote, Physique, V, ch. 3, 227a. 
une relation d'identité entre quelques composants de l'objet de l'intuition et quelques composants l'être vivant.

Pour bien comprendre l'intuition nous avons besoin de considérer la matière et la forme. La nature est matière éternelle en devenir et harmonie ou forme éphémère. Matière que j'appelle «matière-puissance» ou «matière dynamique» (puissance traduit le grec dynamis). La potentialité est la capacité de la matière de devenir tout ce qui existe, aussi bien l'inférieur que le supérieur, du plus simple au plus complexe, raison pour laquelle cette matière n'est pas exactement celle de la science physique. Ensuite la forme est une matière avec des bords plus ou moins stables sans lesquels il n'y aurait pas de processus ni d'entités. Et à cause de son importance je reprends cette idée: dans l'intuition primordiale il y a une identité matérielle et formelle entre quelques composants de l'objet et quelques composants de l'être vivant. La forme et la matière de l'objet laissent en lui leurs traces. Je ne veux pas dire ici par exemple que ce n'est qu'avec une main métallique que l'on peut avoir une intuition d'un objet métallique: je fais allusion au substrat matériel partagé par l'être vivant et l'objet. C'est ensuite au niveau de la pensée consciente symbolique que l'on peut abstraire la forme de la matière.

L'objet et l'être vivant, cela a été dit, sont construits avec les mêmes éléments et les mêmes mécanismes. Cette identité explique la valeur suprême de la métaphore pour le progrès de la connaissance en tant que source de découverte d'affinités. Elle explique aussi le caractère satisfaisant de la connaissance et de la compréhension anthropomorphiques, vérité que chaque être vivant fait sienne à sa façon: l'animal comprend zoologiquement, la plante, phytomorphiquement.

\section{L'HARMONIE DES STRATES NATURELLES}

Le fait que la mécanique, dont l'arrière-plan est géométrique, soit inscrite à la fois dans les objets externes et dans l'organisme animal et humain, explique, je pense, qu'à partir de leur plus jeune âge tous les enfants du monde, scolarisés ou non, et quelle que soit leur culture, aient des intuitions géométriques solides. Il faut conclure que ces intuitions sont ou bien innées ou bien apprises très tôt à mesure que l'enfant appréhende l'espace environnant. Il est donc indéniable qu'il y a une continuité du physique au mathématique, au biologique et au mental, même si aujourd'hui nous ne pouvons décrire cette continuité de façon satisfaisante.

René Thom reconnaît volontiers le caractère énigmatique de ce processus harmonieux qui progresse du physique au mental en passant par le biologique: «il ne fait guère de doute, écrit-il, que la réalisation de la géométrie par des agents physiques comme la lumière ne soit à la source de notre intuition géométrique. . . Comment une structure aussi idéelle que la géométrie peut 
être codée dans notre patrimoine génétique, et parvenir à se réaliser à la fois organiquement — comme dans la morphogenèse de l'œil — et mentalement, c'est bien là un des mystères les plus profonds de la biologie». ${ }^{3}$

\section{L'INTUITION CHEZ LA PLANTE}

Attendu qu'il n'y a pas de conscience sans cerveau et que les plantes n'ont pas de cerveau, il faut écarter d'emblée toute ressemblance entre les plantes d'une part, et le comportement animal et humain d'autre part, quand ce comportement dépend du cerveau. Une plante n'a pas conscience de son existence individuelle. L'intuition végétale n'est jamais consciente, raison pour laquelle les valeurs morales, intellectuelles et esthétiques n'existent pas pour elles ; l'affectivité, le mépris, la dignité, les émotions d'attachement et de rejet, le bonheur et la souffrance non plus. Cela dit, il faut reconnaître chez la plante des comportements inconscients énigmatiques et difficiles à décrire.

Les spécialistes enseignent que la plante perçoit, qu'elle est dotée d'une sorte de vision, d'odorat et de toucher. On sait qu'elle a une intuition de sa propre permanence, permanence qu'elle voudrait préserver, et à cette fin elle utilise convenablement la perception de sa posture et de ses mouvements. La plante est sensible à une série de stimuli tels que la lumière, la température, la gravité, la pression mécanique, la concentration d'une substance chimique, les signaux susceptibles d'être émis par d'autres entités vivantes ou objets inorganiques. Par exemple, étant donné la longueur d'onde de la lumière perçue il est possible de dire, dans un sens à peine figuré, que la plante voit ses voisines et à une distance qui pourrait être supérieure à trois mètres. ${ }^{4}$

Ensuite les plantes s'accommodent à leur présence pour mieux vivre, en s'inclinant ou en modifiant leur rythme de croissance. Elles ont une sorte de mémoire, par exemple, des infections contractées. Comme tout être vivant, elles sont déterminées par le conatus, par le besoin de continuer à vivre, et ce, de la meilleure façon. Attaquées par des insectes elles se défendent en utilisant leurs propriétés chimiques, lesquelles sont également employées pour signaler l'attaque à leurs voisines, qui se protègent en conséquence. La protection contre les attaques et l'envoi de signaux aux autres plantes font penser que la plante assume, pour ainsi dire, que la protection est efficace et que les insectes ne disparaissent pas au moment d'arriver à elle. Ces recherches, bien confirmées, rendent difficile de ne pas reconnaître que dans le comportement de la plante est incarnée et matérialisée inconsciemment une série de notions fondamentales telles que la permanence de l'objet ou

3 René Thom, Apologie du logos, Hachette, Paris, 1990, p. 324.

4 Voir p.ex. Daniel Chamovitz, What a plant knows: A Field Guide to the Sense, Scientific American / Farrar, Straus and Giroux, 2012. 
substance, la causalité, l'uniformité de la nature, l'espace et le temps, autant de catégories qui deviendront tout à fait conscientes et symboliques dans la pensée animale et humaine (nous y reviendrons).

$\mathrm{Au}$ moment de mentionner l'intuition primordiale chez la plante et la façon dont elle matérialise une série de notions fondamentales qui atteindront la strate de la conscience chez les animaux dotés de cerveau, on dira qu'il s'agit simplement d'une vision anthropomorphique et métaphorique de la relation entre la plante et son environnement. Toutefois ici l'anthropomorphisme et la métaphore, tant qu'on n'attribue pas à la plante des propriétés qui n'existent pas sans conscience, sont sources de recherches significatives et de vérités. L'option différente, celle qui nie la continuité et l'harmonie entre les strates naturelles, répugne à la raison. Il convient donc de retenir cette similitude entre les existants: il y a une intuition végétale et animale. C'est l'illustration du fait que la nature n'a pas attendu l'apparition de l'être humain pour commencer à se connaître et à se comprendre.

\section{De L'INTUITION PRIMORDIALE À LA PENSÉE CONSCIENTE ET SYMBOLIQUE}

L'intuition primordiale est la forme la plus primitive, la condition indispensable de toutes les autres formes de la connaissance. Rappelons que l'intuition est inconsciente chez la plante, consciente chez l'animal et l'homme. L'intuition existe parce que l'être vivant est capable d'accueillir la matière et la forme de l'objet. Une telle réception fait penser que pendant ce processus l'être vivant a une attitude passive telle que celle du sable qui conserve les traces des pieds. C'est pourquoi l'intuition primordiale est une sorte de cadeau naturel. Cette passivité de l'être vivant dans l'intuition contraste avec l'attitude active de la conscience au moment de penser symboliquement ainsi qu'au moment d'agir volontairement. Et bien entendu beaucoup de procédures mentales impliquent un mélange de moments actifs et de moments passifs. C'est ce qui arrive par exemple quand nous nous souvenons de quelques événements absents en nous aidant de la perception actuelle.

Le principe de continuité causale, présent dans toute la nature, implique que l'intuition primordiale est un domaine où s'entrelacent des éléments et des propriétés que nous appelons, scientifiquement, physicochimiques, biologiques et psychiques. Cependant ce n'est que chez l'animal et chez l'être humain, parce qu'ils ont un cerveau, qu'existe la potentialité de donner naissance à la pensée consciente et symbolique capable de concevoir et d'imaginer, c'est-àdire de combiner des symboles, en tenant compte y compris d'objets absents.

L'immédiateté de présence de l'objet dans l'intuition explique le fait que celle-ci soit critère infaillible d'existence, d'intelligibilité et de vérité. Dans l'intuition il n'y a jamais de fausseté mais seulement de l'incomplétude: l'intuition est une vérité partielle canalisée, limitée, par les propriétés de 
l'être vivant. Les limites de l'intuition ne sont pas les limites du monde. Contrairement à ce que l'on constate dans l'intuition, dans la pensée consciente l'objet est souvent absent et l'absence est porteuse de faillibilité. L'absence est porteuse de faillibilité (mais au moment d'examiner la pensée consciente nous verrons un autre aspect, éminemment positif, de la capacité de la conscience de se rendre compte d'une absence). Étant donné que l'intuition est la source première du contenu cognitif de l'intellect, la pensée consciente et symbolique, pour acquérir sa portée réelle, intelligible et vraie, doit plonger dans l'intuition.

\section{LA PENSÉE: CARACTÈRE INTELLIGIBLE, OBJECTIF ET ANONYME DES CHOSES}

«Pensée» est un terme multivoque: typiquement sa référence couvre un large spectre qui s'étale depuis la formation des concepts et des propositions jusqu'à l'ensemble des activités de l'appareil psychique humain. Y a-t-il des pensées chez les animaux, les arbres et les pierres ? La réponse dépend de ce que l'on entend par «penser». Il ne me semble pas d'emblée choquant que cette opération, penser, soit concevable de telle façon que les animaux pensent et qu'il y ait des pensées y compris chez les systèmes inorganiques. Comment rendre cette idée admissible?

L'analogie et la métaphore ont été omniprésentes dans l'évolution de la conception symbolique. Le transfert de sens de l'animé à l'inanimé et réciproquement, l'anthropomorphisme aussi, ont été décisifs pour le progrès de la connaissance. Les exemples abondent. «Cause» appartenait à l'origine à la sémantique juridique (j'accuse, il y a un responsable de tel ou tel acte), et le potentiel et la force ont été d'abord la force physique de nos bras.

Les analogies, les métaphores et l'anthropomorphisme n'ont pas de quoi étonner car, après tout, rappelons-le, l'homme, étant naturel, il a été construit avec les mêmes mécanismes employés par la nature ailleurs. Reconnaissons que les animaux pensent et envisageons, au moins pour l'instant, l'existence d'une pensée inorganique. Cela dit, étant donné les différentes conceptions de la pensée, on peut être convaincu que seuls les êtres humains pensent. Ou bien, que seuls les philosophes en sont capables. Et les dualistes du corps et de l'esprit, pour qui penser est une activité spirituelle, diront que personne ne pense vraiment car l'activité spirituelle de l'homme reste encore trop ancrée dans son cerveau.

Bien que nos problèmes ne soient pas, ou ne soient pas principalement une affaire linguistique, il faut donner une idée de ce que l'on entend par «penser» et s'y tenir. Je propose la définition suivante: la «pensée primordiale» est le caractère intelligible, significatif et causal d'une chose. Ce caractère est le substrat invariant dans le passage d'un mode de pensée à un autre et qui assure son uniformité à travers ce passage. Quelque chose est intelligible parce que 
cette chose a un ordre, une raison, une forme harmonieuse, une stabilité, un invariant, des éléments qui existent d'abord en elle et seulement ensuite et d'une façon dérivée dans le psychisme animal et humain. La signification est le prolongement de l'intelligibilité, la marque intelligible que les choses et les processus laissent causalement les uns dans les autres, et la relation causale est la façon dont le caractère intelligible se maintient et se propage. J'appelle «signification causale» le caractère vectoriel de l'intelligibilité, la raison étant que celle-ci agit comme un rayonnement, comme une lumière qui se propage dans son environnement. Il est à remarquer qu'étant donné que la pensée est le caractère intelligible et significatif de quelque chose, la pensée est objective, universelle et anonyme. Ce sont des propriétés héritées par les différents modes de pensée dans les strates supérieures de la hiérarchie naturelle. C'est pourquoi aucune pensée, comme aucune vérité, n'est associée nécessairement au nom d'une personne en particulier.

L'aspect significatif de l'intelligible est la participation d'une chose ou d'un processus au déterminisme causal constitutif de la nature. Cette conception de l'intelligibilité et de la signification implique que toute chose dans l'univers, si elle est réelle, aussi infime soit-elle, est intelligible et significative. Les mécanismes élémentaires du symbolisme existent depuis la matière inanimée. Ensuite, avec l'apparition de la conscience animale et en particulier avec la naissance de la conscience humaine, il y a une augmentation non seulement des caractères intelligibles significatifs mais également du nombre d'éléments susceptibles d'entrer dans la composition de la relation qui constitue la pensée. La pensée primordiale peut exister sans la conscience animale ou humaine. Maintes choses et processus participent au déterminisme causal et sont structurellement stables, possèdent un ordre ou une raison bien avant l'apparition de la conscience, ce qui veut dire - et ce point revêt ici une importance particulière - qu'il y a non seulement des pensées inconscientes telles que celles qui guident le comportement sans que l'on s'en aperçoive, ou qui habitent les rêves, mais qu'il y a aussi des pensées primordiales en dehors de tout cerveau.

On dira que penser est une activité psychique et que mon emploi de «pensée primordiale» est prima facie absurde: quel peut bien être l'intérêt de cet emploi abusif? La pensée est traditionnellement identifiée entre deux ou plusieurs contenus de conscience, par exemple entre un acte de perception et un acte de mémoire ou d'imagination, ou bien, entre un acte de perception et un concept. Cette relation existante dans le psychisme humain se trouve aussi dans le psychisme animal, mais alors pourquoi descendre à des niveaux moins organisés de l'échelle naturelle ? Ma justification est que cette conception de la pensée primordiale est une façon de contribuer à la complétude du champ 
cognitif unique et sans ruptures, image d'une réalité dépourvue de nouveautés abruptes.

La pensée primordiale structure l'uniformité du développement de la pensée à tous les niveaux, de l'inorganique jusqu'à l'être humain. Rappelons qu'au niveau inorganique les choses sont intelligibles et significatives parce qu'elles sont causalement déterminés. La matière inanimée est également la strate où fait son apparition, de façon primitive, la syntaxe. La condition de l'acceptation de cette affirmation est qu'à ce niveau la syntaxe soit conçue comme l'ensemble des règles de combinaison des pensées primordiales. Dans cette strate inorganique ces règles sont les lois de la physique et de la chimie. Considérez par exemple les formules chimiques représentatives des éléments formateurs d'un composé et la proportion dans laquelle ils se combinent.

Le statut ontologique de la pensée primordiale au niveau inorganique et végétal est physique et chimique, et ici la pensée primordiale est l'influence causale physique ou physicochimique exercée par un objet sur un autre. Néanmoins au fur et à mesure qu'on monte dans l'échelle naturelle vers la strate psychique et sociale des animaux supérieurs et de l'homme, le statut ontologique de la pensée devient plus difficile à établir. La raison en est notre ignorance concernant le rapport entre le cérébral et le mental, reflétée dans la façon ontologiquement dualiste de les décrire. Et en effet un leitmotiv de mon naturalisme est l'affirmation selon laquelle nous n'avons pas les concepts appropriés ni pour décrire ni pour expliquer la dynamique entre ce que nous appelons aujourd'hui le physique et le mental, ni pour décrire ni pour expliquer le contrôle mutuel entre eux. Il s'ensuit que nous ne sommes pas au bout de nos peines car même l'affirmation selon laquelle la pensée primordiale au niveau inorganique est ontologiquement physique n'est nullement autorisée par les formalismes et les procédures de la physique actuelle. Pensez aux expériences somatopsychiques ou psychosomatiques: le stress qui entraine la fatigue, le mot qui fait rougir révèlent une intégration, une identification du physique et du mental non descriptible par la sémantique et les procédures de la physicochimie actuelle, ce qui ne veut pas dire que les sciences ne puissent pas améliorer leurs abstractions.

\section{LA PENSÉE DANS LA STRATE DES ANIMAUX SUPÉRIEURS}

Tandis que le végétal obtient son énergie par photosynthèse, l'animal l'obtient par prédation. C'est principalement dans sa fonction physiologique que le végétal se différencie de l'animal et ce fait a des conséquences dans leurs manières respectives d'apprécier l'intelligibilité et la signification. L'animal supérieur est un être doué d'une conscience qui lui permet d'avoir une représentation de son environnement et de se rendre compte que dans cet environnement il est un centre d'appréciation de son milieu. Concernant son 
rapport à la vie végétale, l'animal supérieur présente une répétition et une nouveauté. Ainsi chez l'animal, comme chez le végétal, l'unité de pensée est le caractère intelligible et significatif des processus ou des entités, et le critère permettant de détecter la valeur de la pensée pour l'animal reste le même que pour les végétaux: la réaction face aux propriétés de l'environnement. La nouveauté est, naturellement, l'apparition de la conscience.

Les Stoïciens ont nommé conscience la connaissance de la connaissance, ce tact intérieur qui permet à chacun de dire je ou moi. La pensée consciente est, pour ainsi dire, une pensée de second ordre, une pensée d'une pensée. Par exemple l'expérience de la douleur est un caractère intelligible et significatif du monde, tandis que la pensée consciente de ma douleur n'est pas elle-même douloureuse, mais elle peut moduler l'intensité perçue de la douleur. La conscience permet à l'organisme de se rendre compte de ses limites: certaines choses sont connues, d'autres, ignorées. Elle nous permet de nous rendre compte non seulement de la présence des choses mais aussi de leur absence. Cette propriété consistant à nous rendre compte d'une absence présuppose, en plus de la perception, la mémoire et l'imagination, facultés dont les effets peuvent être positifs. D'autre part, tout être conscient vit le fait que cette capacité de se rendre compte d'une absence est, aussi, dramatique.

En effet, une façon paradigmatique qu'ont les animaux supérieurs de manifester individuellement des états de conscience est leur capacité à remarquer une absence. Pensez à la tristesse de l'animal de compagnie suite à la disparition de son maître. Par ailleurs, en cohérence avec l'impossibilité de concevoir des nouveautés absolues, il n'est pas tout à fait insensé de se demander, par exemple, si les collectivités d'insectes ne possèdent pas elles aussi, en tant que collectivité, une certaine forme de conscience primitive. Certaines colonies de fourmis, pendant la construction de la fourmilière, se rendent compte que les champignons qui les alimentent manquent de feuilles pour se nourrir, et elles se chargent donc de leur en apporter. On observe le même comportement, i.e. évaluation de la situation, décision de l'améliorer d'une façon optimale ou presque optimale, chez les abeilles: se rendant compte qu'il n'y a pas suffisamment de miel accumulé et calculant à leur façon ce qui manque, un nombre convenable d'entre elles sort de la ruche pour chercher le nécessaire.

L'ordre causal des caractères intelligibles et significatifs se trouve encapsulé dans l'uniformité typique de la pensée: à la succession causale réelle correspond l'ordre syntaxique et logique avec lequel l'animal structure ses pensées. Ce que l'animal imagine syntaxiquement et logiquement correspond par exemple à l'ordre causal du fait que le prédateur affamé attrape et tue sa proie. Sa pensée ainsi structurée lui permet d'anticiper les faits et à l'animal en tant que proie de se mettre opportunément à l'abri. L'emploi de 
l'indice ou du symbole, le cri ou le geste typique lui permettent d'alerter ses congénères de la présence ou de l'arrivée d'un prédateur.

Il importe de bien se rendre compte de ces deux faits: premièrement, la conscience ne crée ni le caractère intelligible ni la signification car autrement il n'y aurait pas d'uniformité entre les pensées végétales et animales ; deuxièmement, une fois que l'animal héberge les pensées dans sa subjectivité ou intériorité, les pensées sont objectivées et déclarées entités existantes. Sans l'objectivité de la pensée consciente le monde serait la création de la conscience. Il serait dans le mot. Un rêve qui déterminerait notre activité. C'est ce qui arrive aux malheureux animaux à qui on détruit les mécanismes cérébraux inhibiteurs de l'action motrice lors du sommeil. En effet, des chats à qui on détruit la partie caudale du locus corruleus peuvent poursuivre des souris imaginaires pendant qu'ils rêvent, ou prendre refuge, angoissés, devant les prédateurs de leurs cauchemars.

Le psychisme agit comme un filtre: bien que dans la nature il y ait un nombre indéfini de pensées primordiales, seulement quelques-unes sont reconnues. L'opération de la conscience est conditionnée par les caractéristiques biologiques et psychiques de l'organisme, par la finesse des organes de perception. En vue de satisfaire le besoin de vivre et de continuer à vivre, l'évolution a déterminé ou programmé l'animal biochimiquement et géométriquement pour qu'il puisse reconnaître, dans l'espace et dans le temps, la nourriture et le partenaire sexuel, les congénères et les prédateurs, le couple pour la reproduction, les endroits dangereux et les sites accueillants pour le repos. Reconnaître ces entités et avoir envers elles le comportement approprié, signifie que l'animal a saisi correctement la participation de telles entités dans le réseau causal. Ces entités ou processus - la permanence de l'objet, l'espace, le temps, la nourriture, le congénère, le prédateur, le couple pour la reproduction - sont autant de catégories, de pensées, de caractères intelligibles doués de la signification suprême pour l'animal: leur valeur vitale.

La fonction de la conscience animale ne se réduit pas à saisir les unités intelligibles mentionnées et à se les représenter correctement. Le comportement de l'animal rend manifeste qu'il combine les pensées, et la combinatoire est une logique et un ensemble d'algorithmes, une activité intérieure ordonnatrice. Cette activité signifie une expansion consciente de l'esquisse primitive de syntaxe et de logique qui existe ailleurs dans des formes de vie non conscientes où l'ordre syntaxique et logique est invariablement une représentation de l'ordre causal des événements. Il est sensé de voir dans cette activité subjective ordonnatrice à la fois l'expression d'une exigence organique et les marques laissées dans l'organisme par l'ordre des choses externes. Pendant que l'être vivant vit, pour ainsi dire, normalement, il y a circularité et harmonie entre les propriétés de l'organisme, les exigences qu'il doit satisfaire pour vivre, les 
propriétés intelligibles du milieu et l'ordre causal des entités et des processus (les pensées primordiales). Sans cette harmonie l'organisme disparaît. À la structure ordonnée composée de propriétés intelligibles et de relations causales entre des entités et des processus correspond, dans le psychisme animal, la syntaxe et la logique, indispensables à la formation de propositions et de raisonnements.

Ce n'est pas tout: indispensable à la pensée animale et humaine est aussi l'emploi de symboles. Le symbole, étant une expansion de l'intuition primordiale, de l'image et de l'indice, possède des antécédents distincts non seulement dans la strate organique inférieure à celle de l'animal mais aussi dans la strate de la matière inorganique. C'est la raison pour laquelle la pensée, en tant qu'activité intellectuelle symbolique, ne signifie pas non plus une nouveauté abrupte par rapport à ce qui existe dans le reste de la nature. On a tendance à considérer qu'il y a entre le symbole et le symbolisé une relation conventionnelle. Or cette vision, bien qu'elle comporte une part de vérité plus ou moins grande selon les cas, est inintéressante. Il importe, par contre, de bien se rendre compte que le symbole est souvent une analogie, une métaphore, une synthèse d'idées dont les racines sont réelles et objectives, et, en conséquence, instructives: c'est le sens pertinent dans le contexte présent.

Le cri d'alarme pour attirer l'attention de ses congénères émis par l'animal qui repère un prédateur prouve que les animaux sont capables de développer et d'employer des symboles objectifs: le cri a la même signification tout à fait objective, indépendamment de l'individu émetteur et de l'état subjectif dans lequel il se trouve. Ce genre de communication rend manifeste qu'un symbole tel que le cri d'alarme présuppose la vie en société et la sensibilité des animaux à la vie sociale. Sans cette présupposition un animal pourrait éviter l'emploi de symboles et se comporter d'une manière égoïste.

\section{De L'ANIMAL À L'HOMME}

Quel contraste entre le mécanisme, simple et élémentaire, de la réaction du tournesol face à ce qui est intelligible et significatif pour lui, et la complexe dynamique constitutive du raisonnement animal et, a fortiori, de la pensée humaine. Quand l'être humain pense, il lui arrive de vivre l'insuffisance des systèmes de symboles, des lieux communs, des idées reçues. Il voit sa pensée aplatie. Il a cette expérience quand sa perception est fine et son intuition profonde, quand son exigence de précision concrète est élevée. Il est probable que de temps en temps l'animal ait, lui aussi, mais dans une moindre mesure, la sensation que ses moyens d'expression sont en deçà de ce qu'il faudrait pour s'exprimer comme il le voudrait.

J'ai rappelé à plusieurs reprises la remarquable capacité de la conscience à se rendre compte de l'absence de quelque chose. Ainsi, à sa façon, elle rend 
présent l'absent. Sur ce point il semblerait qu'il y ait une différence de degré entre l'homme et l'animal. Pour changer une ampoule au plafond (que je n'arrive pas à saisir) je mets en correspondance, imaginativement, ce que je vois: l'ampoule, son emplacement et mon corps, avec ce que je ne vois pas: une chaise ou un escabeau, et je vais chercher l'une ou l'autre. Par contre les singes de Kœhler, attirés par une banane au-delà de leur portée, ne semblent voir l'utilité de la grosse caisse pour l'atteindre que si elle est vue en même temps que la banane.

Aussi bien chez l'animal que chez l'être humain, la pensée consciente symbolique est une dynamique interne plus ou moins complexe qui permet l'introduction d'une distance entre les stimuli et l'action ou réaction de l'organisme. La complexité et la distance sont mesurables par une série de facteurs: par le nombre de caractères intelligibles et significatifs auxquels l'animal ou l'homme sont sensibles; par le nombre de symboles qu'ils sont capables d'utiliser et, s'agissant de l'homme, par la richesse de son langage ; par l'étendue du champ enveloppé par l'espace et par le temps ainsi que par la profondeur et la portée des relations causales que l'animal ou l'homme sont capables de connaître. Cet instrument pour mesurer la distance entre les stimuli et l'action de l'organisme rend manifeste, premièrement, que la distance introduite par la pensée humaine est supérieure à celle dont 1'animal est capable. Deuxièmement, il fait comprendre que la distance entre le moi qui agit d'un côté, et le moi qui pense sur son action et l'évalue, d'autre part, est, elle aussi, supérieure chez l'homme à celle dont l'animal est capable.

La distance entre les stimuli et les réponses introduite par la complexe dynamique mentale aussi bien chez l'être humain que chez l'animal est la source à la fois du progrès de la connaissance et de l'erreur. La richesse de son langage, le fait de nommer les objets permet à la personne de prendre un certain recul face aux exigences biologiques auxquelles les animaux semblent être davantage attachés. Cette distance permet l'épanouissement de l'être humain, le développement de sa pensée sous forme d'art, de science et, en particulier, sous forme de philosophie. Sans recul par rapport à une réalité, quelle qu'elle soit, point de méditation.

\section{CONSCIENCE D'UNE PRÉSENCE ET D'UNE ABSENCE: GRANDEUR ET LIMITES DE LA PENSÉE SYMBOLIQUE}

La faculté suprême de la pensée consciente symbolique est, je l'ai dit, le pouvoir de l'animal et de l'être humain de se rendre compte d'une présence et d'une absence, avec tout ce que cela comporte de dramatique et parfois de positif. C'est pourquoi pour comprendre le passage de l'intuition primordiale à la pensée consciente symbolique il faut comprendre non seulement comment l'être vivant arrive à se rendre compte d'une présence par l'intermédiaire de 
son appareil perceptif, mais également comment il arrive à se rendre compte d'une absence, c'est-à-dire à imaginer l'inexistant. Thèse sur l'imagination: elle n'est pas une faculté de la conscience. Elle est sa capacité de ne pas s'arrêter dans aucune de ses activités: elle va au-delà de la perception grâce au souvenir, au-delà de la perception et du souvenir conduite par l'affectivité ; la conscience va au-delà de ses activités psychiques grâce aux mécanismes des systèmes de symboles. Cette capacité de ne pas s'arrêter n'est ni libre ni spontanée: elle est causalement canalisée par les actes conscients différents de l'opération dépassée.

Tout comme dans l'arbre les éléments les plus conditionnés parce qu'ils émergent en dernier sont la fleur et le fruit, ainsi la pleine conscience, étant la dernière dimension qui émerge dans l'organisme animal et humain, est son pouvoir le plus conditionné. La conscience est conditionnée principalement par l'ensemble de causes qui la produisent et par la nécessité de continuer à exister. Dans les mécanismes biochimiques de l'intuition existe, en puissance, la signification qui sera symboliquement articulée par la pensée. Attendu que la nature est un réseau serré de causes multiples et variées, elle est, nous l'avons dit, continue, et cette continuité impose la vision selon laquelle, primo, l'intuition est une région où s'entrelacent causalement le mathématique, le physicochimique et la matière vivante, secundo, de l'intuition émerge, à son tour, la pensée consciente symbolique.

Attendu que la conscience est conditionnée par le conatus, par la nécessité de continuer à exister, je propose l'hypothèse selon laquelle la pensée consciente symbolique se développe, essentiellement bien que non exclusivement, chaque fois que l'intuition ne suffit pas à assurer notre besoin de survie.

Voici, pour terminer, ma liste des catégories. Son explication et justification devra attendre une autre occasion, cette fois je me borne à les mentionner. Ce sont des concepts sans lesquels aucun être vivant ne pourrait vivre. La catégorie mère est (I) la matière éternelle et continue. Cette matière est une source inépuisable de formes, d'énigmes et de surprises. Les autres concepts fondamentaux en sont des aspects. La matière éternelle est causalement ordonnée, ce qui implique (II) la catégorie d'ordre causal, laquelle, à son tour, implique (III) le concept fondamental de nécessité. La matière éternelle, causalement ordonnée, est la substance qui se manifeste par (IV) des formes plus ou moins stables dans (V) l'espace et dans (VI) le temps. Ainsi, toute forme naturelle est matérielle, et contrairement à une croyance assez répandue depuis les modernes et jusqu'à aujourd'hui, l'espace et le temps ne sont pas des substances rivales de la matière éternelle mais plutôt, comme Aristote à sa façon les avait conçus, des aspects de la matière (hylê). Ce sont ces catégories qui ont guidé l'épanouissement de cet essai et notamment le passage de 
l'intuition primordiale à la pensée symbolique.

\section{RÉFÉRENCES BIBLIOGRAPHIQUES}

ARISTOTE, Physique, texte établi et traduit par Henri Carteron, Les Belles Lettres, Paris, 1990.

CHAMOVITZ, Daniel, What a plant knows: A Field Guide to the Sense, Scientific American / Farrar, Straus and Giroux, 2012.

ESPINOZA, Miguel Espinoza, A Theory of Intelligibility. A Contribution to the Revival of the Philosophy of Nature, Thombooks Press, Toronto, ON, 2020. , Pensar la naturaleza. Epistolario filosófico, Biblioteca Magna, Uniediciones, Bogotá, 2020.

LARGEAULT, Jean, Principes de philosophie réaliste, Klincksieck, Paris, 1985. , Intuition et intuitionisme, Vrin, Paris, 1993.

THOM, René, Apologie du logos, Hachette, Paris, 1990, p. 324. , Esquisse d'une sémiophysique. Physique aristotélicienne et Théorie des catastrophes, Inter Éditions, Paris, 1988.

Miguel Espinoza es Profesor de Filosofía de la Naturaleza y de Filosofía de las Ciencias en la Université de Strasbourg.

Lineas de investigación

Filosofía de la Naturaleza, Filosofía de las Matemáticas y de la Física

Publicaciones recientes

2014: Repenser la naturalisme. Paris: L'Harmattan, 2014.

2017: La matière éternelle et ses harmonies éphémères, Paris: L'Harmattan, 2017.

Correo electrónico: miguel.a.espinoza.v@gmail.com 
